\title{
Modularized and Navigated Mobile Shopping Assistance System
}

\author{
Rajani Thite \\ Department of Information \\ Technology \\ DYPCOE,AMBI,TALEGAON
}

\author{
Mahesh Dangale \\ Department of Information \\ Technology \\ DYPIET,AMBI,TALEGAON
}

\author{
Prashant Kumar \\ Department of Information \\ Technology \\ DYPIET,AMBI,TALEGAON
}

\author{
Kunal Rathod \\ Department of Information Technology \\ DYPIET,AMBI,TALEGAON
}

\author{
Nilesh Gupta \\ Department of Information Technology \\ DYPIET,AMBI,TALEGAON
}

\begin{abstract}
This framework is settled to solve the problems of the customers while shopping. i.e. problems to find the products and the location of a particular the latest info and promotions, Modularized and Navigated Mobile Shopping System Assistance System which will be supported by various mobile technologies such as wireless network, GPS, NFC etc. and a safe encryption for providing safe and protected task to ease the shopping process
\end{abstract}

\section{General Terms}

Recognition, Security, Algorithms

\section{Keywords}

NFC, Shopping assistance system, Mobile shopping, Modular shopping, secure payment, Mobile shopping Assistance.

\section{INTRODUCTION}

A customer shopping task consists of some necessary and optional tasks like payment or information recovery. This paper presents a general idea of the possible solutions in the field of Mobile Communication that can be applied in order to perform these tasks in future applications based on current and forthcoming communication and collaboration technologies. The tasks are symbolized by several modules, like shopping list management, orientation, shopping cart management, coupons redemption and payment. These components conceptually describe the available opportunities to recognize specific parts of a customer shopping process based on several technologies and customer activities. In particular, a It has a significant advantage for the vendor as it enables the analysis of the benefits and drawbacks of technologies and services. Furthermore, it allows the investigation independent from basic technologies like RFID and barcodes. Irrespective of the used technology, the modules can be allied with each other which allow a simple combination of several modules in a vendor's organization. Depending on the usage context, several groupings of modules are more valid than others

\section{Modules}

1. Shopping list management

2. Orientation

3. Product information

4. Shopping Cart management

5. Coupon redemption
6. Customer loyalty points

7. Payment

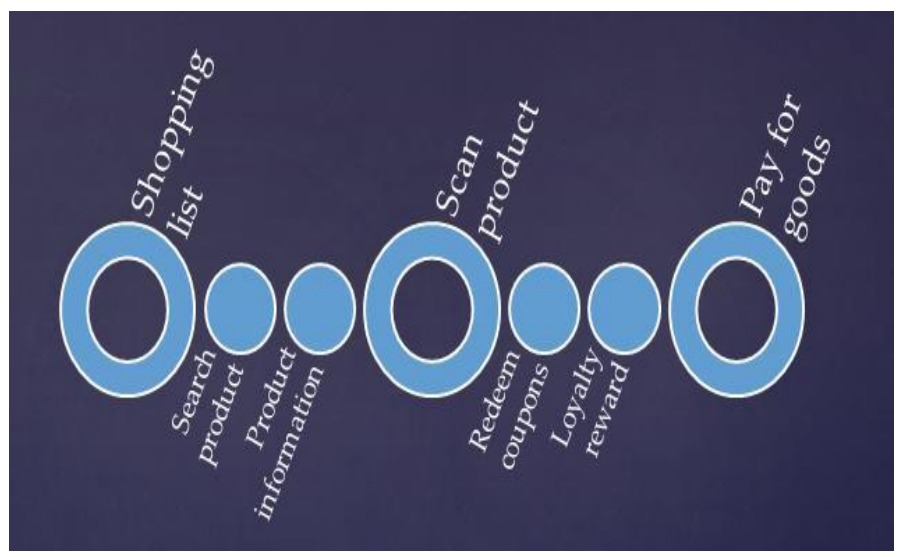

Fig. Process Workflow

People love shopping and their mobile phones. They carry phones everywhere they go. So, why not use mobile instead of other stuff like shopping card, credit card and shopping list. There are some application that can perform some individual tasks like shopping list management and payment. But they cannot fulfill the requirements of retailer's infrastructure. This system can be changed /upgraded easily. More secure ways for payment system.

\section{IMPLEMENTATION ASPECTS OF PROPOSED MODEL}

A) Mobile based Shopping Assistance System.

This method summarizes the work on design and operation of Mobile based Modularized Shopping Assistance System. The system consists of three main modules:

1. Adding products to shopping catalogue.

2. Finding the products in the store

3. Coupons redemption and final payment.

B) Is the process feasible and secure? As far as feasibility is concerned, it is of two types: 1.Procedure feasibility, 2.Technical feasibility. Procedure 
Feasibility will consist of the algorithms which are being used in the system development. These are: A* Algorithm, XOR Encryption-Decryption Algorithm for security purpose. Technical feasibility mentions to the technologies used to transmit the system workflow.

Table 1: Technical feasibility

\begin{tabular}{|c|c|}
\hline Programming Language & JavaScript, PHP \\
\hline Hardware & NFC Smartphone \\
\hline Front End Programming & JAVA \\
\hline Technology & NFC, Wireless Network \\
& \\
\hline
\end{tabular}

\section{PROJECT WORKFLOW}

The projected planning helps in understanding Modularized and Navigated Mobile Shopping Assistance System. The modules of the following workflow are: Registration flow, Product Management flow, Purchase Process flow, Database flow. The explanations of the above named components are as follows:

\subsection{Registration Flow}

To purchase products at the market, the customer firstly has to create a user account and should provide all the required information. The information provided will be stored in the databases of the market.

\subsection{Product Management Flow}

Supplier has to put all the information of available products including id for each product with the corresponding barcode of that product. Products can be equipped with NFC tags for more comfort.

\subsection{Purchase Process Flow}

a) Firstly, the Customer has to make a shopping catalogue and select the products to purchase either using any of the existing mobile technology, his shopping catalogue will be saved in his local and web databases.

b) Customers will be suggested new products as per their interest and earlier purchases and the popularity of that product.

c) Customer will be navigated to the product with the help of shopping store's map and customer current location (using algorithm 1).

d) Customer pay for purchased products based on his shopping catalogue using NFC service. The billing information is protected using XOR encryption algorithm (using algorithm 2).

e) After purchasing, the purchased products will be stored in Customer account and those products will also be removed from the Seller's database. NFC tags will be removed after the purchase of that product.

\section{4. ALGORITHMS}

\section{1.) A* Algorithm}

A* is a computer algorithm that is widely used in path finding and graph traversal. The algorithm uses the heuristic function to find out the goal node from the initial node. This algorithm to plot a path to the products in the shopping mall.

Function $A *($ start, goal)

closedset : = the empty set // set of already evaluated nodes.

openset $:=\{$ start $\} \quad / /$ The set of tentative nodes to be evaluated, at first containing the start node

came_from := the empty map // navigated nodes map.

g_score[start] $:=0 \quad / /$ Cost from start along best known path.

// Estimated total cost from start to destination through $y$.

$f \_s c o r e\left[\right.$ start] $:=g \_s c o r e[$ start $]+$ heuristic_cost_estimate(start, goal)

whileopenset is not empty

current $:=$ the node in openset having the lowest f_score[] value

if current $=$ goal

returnreconstruct_path(came_from, goal)

remove current from openset

add current to closedset

\section{2.) XOR Encrypt Decrypt Algorithm}

Encryption and Decryption algorithms are used to transform a plain text into one that is unintelligible, and then again back to its original form it is generally done to provide security to the user's confidential information.

Encryption Algorithm:

1. $k=$ encryption key.

2. $t=$ to encrypt text.

3. res $=\{$ empty $\}$

4. Loop (length of $t$ )

$4.1 \mathrm{cc}=$ character code of current char of $t$.

4.2 res $=$ res + to character from code $(k X O R c c)$.

5. End

Decryption Algorithm:

1. $k=$ encryption key .

2. $t=$ to decrypt text.

3. $r e s=\{$ empty $\}$

4. Loop (length of $t$ )

$4.1 c c=$ character code of current char of $t$.

4.2 res $=$ res + to character from code $(k X O R c c)$.

5. End

Time complexity $=O(n)$. 


\section{CONCLUSION}

In this framework, the Customer Shopping process have been mapped to modules. These modules consist of Shopping Catalogue management, Orientation, Product information procurement, Shopping Cart management, Coupon Redemption, Customer Loyalty Points and Payment. Each module is being designed and implemented using a set of different mobile technologies. As the modules are selfgoverning, it is probable for the seller to implement a solution which could satisfy the market's needs. For example, it can merge scanning of barcodes on products for the shopping cart management and NFC technology for the payment process. The general idea of the modules including the available technologies are intended of helping seller to identify possible advantages or disadvantages of technologies or services for their stores. The retailers can easily integrate the modules in their infrastructure after deciding which modules they want to implement and which technology they want to use.

Even in the future if the available technologies get changed, e.g. through the combination of sensors into the shopping atmosphere, which can e.g. recognize the customer and his behavior, the seven modules will be retained, just the technology and the customer's behavior will change. It is intended to implement all defined components including all mentioned technologies and use cases. Using this shopping framework, numerous studies will be conducted which shall identify which technologies and use cases customers used to prefer for their shopping process.

\section{ACKNOWLEDGEMENTS}

We would like to express our sincere gratitude for the assistance and support of a number of people who helped us in our project.

We are grateful to our internal guide Prof. Rajani Thite, Department of Information Technology, Dr. D. Y. Patil College of Engineering, for providing her valuable guidance and constant supervision at various stages throughout the project work. She has been a source of motivation enabling us to give our best efforts in this project. We are also grateful to Prof. Ravi Patki, Head of Information Technology Department, Dr. D. Y. Patil College of Engineering and other staff members for encouraging us and providing all possible help, assistance and facilities.

\section{REFERENCES}

[1] Modularization of mobile shopping assistance systems by Paradowski, Denise; German Research Center for Artificial Intelligence Campus D3_2, 66123 Saarbrücken, Germany ; Kruger, Antonio.

[2] An NFC-Based Solution for Discount and Loyalty Mobile Coupons by Sanchez-Silos, J.J.; Dept. of Computer. \& Numerical Anal., Univ. of Cordoba, Cordoba, Spain

[3] Mobile Near Field Communications (NFC) "Tap " $n$ Go" Keep it Secure \& Private By Ann Cavoukian, Ph.D Information and Privacy Commissioner, Ontario, Canada

[4] Overill, R.E., Review: Foundations of Cryptography, Volume II: Basic Applications. Journal of Logic and Computation, 2005. 15(3): p. 405-405.

[5] L. Chen and G. Gong, Communication System Security., 2012, CRC Press

[6] Importance Measures for a Modular Software System by Fiondella, L. ; Dept. of Computer. Sci. \& Eng., Connecticut Univ., Storrs, CT ; Gokhale ,S.S.

[7] Mobile Remote Presence Systems for Older Adults: Acceptance, Benefits, and Concerns, 2011, ACM Press

[8] T. Kanda, "A communication robot in a shopping mall", Robotics, IEEE Transactions on, vol. 26, no. 5, pp. 897-913, 2010

[9] T. Imielinski and B. R. Badrinath, "Mobile Wireless Computing in Data Management", CACM, pp. 18-29, 1994 